\title{
Green's function based space-charge field solver for electron source simulations
}

\author{
Mark Hess, Chong Shik Park, and Daniel Bolton \\ Indiana University Cyclotron Facility, Bloomington, Indiana 47408, USA
}

(Received 21 November 2006; published 17 May 2007)

\begin{abstract}
We present a theoretical formulation for calculating the electromagnetic space-charge fields within a simplified electron source geometry using time-dependent Green's function methods. The source geometry is assumed to be comprised of a flat cathode along with a pipe of arbitrary but uniform cross section. Under the assumption that the beam currents are parallel to the pipe axis, we derive exact solutions for the electromagnetic potentials in the Lorentz gauge. In addition, for the special case of a pipe with rectangular cross section, we present the exact solutions of the electromagnetic potentials for arbitrary beam currents. Finally, we show the results of an analytical benchmark study in which the electromagnetic fields that are solved using the Green's function method are in excellent agreement $(<1 \%$ error) with the benchmark fields.
\end{abstract}

DOI: 10.1103/PhysRevSTAB.10.054201

PACS numbers: 41.75. $-\mathrm{i}, 52.59 . \mathrm{Sa}$

\section{INTRODUCTION}

Modern high-energy electron accelerators require electron sources, such as photocathode sources, that produce high-brightness, space-charge dominated beams [1]. Since the electron beam is typically nonrelativistic near the source cathode, it has a low relativistic mass factor which makes space-charge forces significant. In order to predict relevant beam parameters at the exit of the source, such as the beam emittance, it is essential that the space-charge fields be accurately computed.

There are a number of important effects within an electron source which suggest that a fully self-consistent electromagnetic theory is necessary to describe the spacecharge fields. For example, in the Brookhaven National Laboratory $2.856 \mathrm{GHz}$ rf photocathode source [2], the beam which is generated by a laser pulse is initially bunched with a bunch length that is small compared to the wavelength of the rf electric field. Moreover, the bunch is rapidly accelerated from nonrelativistic energies near the cathode, to relativistic speeds of order $0.8 c-0.9 c$ within a quarter wavelength of the rf field. Both of these properties imply that the space-charge fields within an rf photocathode source will be highly time dependent, and the effects of causality can be extremely important. In addition, these sources contain metallic conducting surfaces, such as a cathode, side walls, and irises, which impose nontrivial boundary conditions on the space-charge fields. Qualitatively, any charge near a flat metallic cathode will give rise to an "image" charge, but the more complicated boundary conditions such as curved side walls and irises will yield complicated wave reflections within the system. Hence, in order to completely model the physics of modern electron sources, it is necessary to have a self-consistent electromagnetic field solver, which computes the spacecharge fields in the presence of conducting boundaries. We note that the space-charge fields which we discuss in this paper are the electromagnetic fields that are generated by the beam in the presence of the conducting boundary conditions of the electron source. In other words, the space-charge fields which we derive include the effects of the image charges and image currents on the conductor boundary of the source. The surface of the electron source is assumed to be perfectly conducting, i.e., the parallel electric and the perpendicular magnetic fields at the surface of the source are assumed to be zero.

The accelerator physics community has developed a wide range of simulation codes that compute space-charge fields in a variety of ways. The widely used workhorse code PARMELA [3] can simulate beam dynamics within a source for arbitrary external electric and magnetic fields, but under the assumption that the space-charge fields are electrostatic. TREDI [4], a fully electromagnetic code, computes the exact space-charge fields using Lienard-Wiechert potentials. However, TREDI at present can only model the presence of a flat cathode with no other conductor boundaries present.

Fully electromagnetic particle-in-cell (PIC) codes, such as MAFIA [5], can calculate space-charge fields in the presence of arbitrary conducting boundary conditions by using grid based field solving methods, such as Yee's algorithm [6]. However, simulations of electron sources with PIC codes can incur two difficulties. First, since the beam bunch length is small, the longitudinal cell length in the region near the bunch needs to be sufficiently short. Moreover, the initial electromagnetic fields which are generated when the bunch is emitted at the cathode will have a spatial size that is comparable to the bunch length. Since these fields propagate spherically away from the bunch to other points in space, it is necessary to maintain a sufficiently small grid size everywhere in space. This typically requires a large number of cells and intensive computational requirements for the PIC algorithm. The second difficulty arises from the fact that solving the fields of a beam bunch on a grid results in unphysical properties, such as numerical grid dispersion [7]. This implies that the 
phase velocity of an electromagnetic wave within the simulation is a function of the angle of propagation relative to the grid. Numerical grid dispersion can lead to unwanted effects in beam simulations, such as numerical Cherenkov radiation.

Recently, there have been analytical methods developed for calculating the space-charge fields in a photocathode source in the presence of a conducting cathode and circular pipe $[8,9]$. These methods expand the potentials using a Fourier transform for the longitudinal direction and a Bessel function expansion in the transverse direction. In Ref. [8], the fields were computed from the electromagnetic potentials in the Lorentz gauge under the assumption of uniform density bunches and uniform bunch acceleration in the longitudinal direction. The main advantage of these techniques is that it is possible to calculate the spacecharge fields to arbitrary accuracy for a given beam charge and current density when a sufficient amount of eigenfunction modes and Fourier $k$-space are included.

In this paper, we develop a similar approach as Ref. [8] of constructing the electromagnetic potentials using the Lorentz gauge for a flat cathode and a pipe with an arbitrary cross section that is axially uniform. For arbitrary longitudinal beam currents, we construct the electromagnetic potentials in this system using time-dependent Green's functions. The Green's functions are expressed in a compact form such that only integration in real space-time is required. The Green's function also features explicit causality constraints, which allow for highly accurate computation of interesting effects, such as the physics near the head of the bunch when the bunch is emitted from the cathode. Since Green's functions are generated by delta function sources, it is possible to accurately compute the space fields of electron bunches with arbitrary bunch length. In addition, since the electromagnetic fields which are derived in the Green's function formalism are defined everywhere in space, as opposed to a grid based definition in a PIC code, the electromagnetic fields will be dispersion free. We also show the more general solution for the electromagnetic potentials when transverse currents are present in the special case of a rectangular pipe. Finally, we show the results of a benchmark study in which the fields computed within the Green's function based algorithm are compared to those derived from an analytical result. The difference between the fields in both cases is less than $1 \%$.

Our paper is organized as follows. In Sec. II, the 3D electromagnetic potentials are developed analytically for the two cases: (a) a pipe which is axially uniform with an arbitrary cross section that contains longitudinal beam currents and (b) a rectangular pipe with arbitrary beam currents. In Sec. II A, we give explicit solutions for the case of a circular pipe. In Sec. III, we compare the results of the Green's function based field solver with an analytical benchmark case. In Sec. IV, we give a summary of our results.

\section{FORMULATION OF ELECTROMAGNETIC POTENTIALS AND FIELDS}

The electron source that we consider consists of two perfectly conducting components: a flat cathode which is located at $z=0$ and a surrounding pipe of arbitrary uniform cross section that extends to infinity in the positive $z$-direction. In addition, the source contains an electron beam with a charge density $\rho(\mathbf{r}, t)$ and a current density $\mathbf{J}(\mathbf{r}, t)$. Figure 1 shows a schematic of the source. In this section, we will show the solutions to the electromagnetic space-charge potentials for the electron source in two specific cases. First, we will show the solutions to the potentials and fields for a pipe which is axially uniform but has arbitrary cross section, assuming that the beam currents are longitudinal, i.e. $\mathbf{J}(\mathbf{r}, t)=J_{z}(\mathbf{r}, t) \hat{\mathbf{e}}_{z}$. As an example, we will present the potentials for a pipe with a circular cross section. Second, we will show the solutions to the electromagnetic potentials for a pipe with a rectangular cross section, under the assumption that the beam currents are arbitrary. Although most accelerator systems do not incorporate electron sources of rectangular cross section, the second case is of theoretical interest since it represents a completely solvable system for general beam currents. The solutions to the electromagnetic potentials for the most general problem of arbitrary beam currents within a pipe of arbitrary, but uniform, cross section have not yet been solved. We note that the assumption of the beam current being solely in the longitudinal direction is actually a good approximation for computing the spacecharge fields in most photoinjector systems. This approximation is justified by comparing the ratio of the transverse beam velocity to the longitudinal beam velocity. When the beam is nonrelativistic near the cathode, the velocity ratio

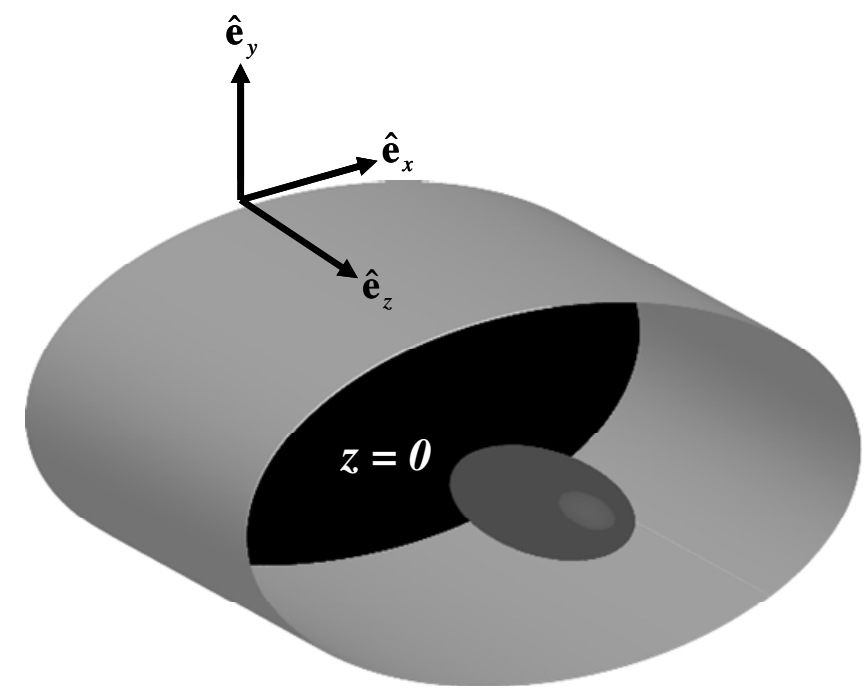

FIG. 1. Schematic of an electron source showing a planar cathode at $z=0$, a surrounding pipe which has an arbitrary cross section but is axially uniform for $z>0$, and an accelerating electron bunch. 
is of the order $\left|v_{\perp} / v_{z}\right| \sim\left|E_{\perp} / E_{z}\right|$. The longitudinal component of the electric field, $E_{z}$, is approximately given by the external rf electric field, and the transverse component of the electric field, $E_{\perp}$, is due to both the transverse rf electric field, as well as the transverse space-charge electric field. For the BNL $2.856 \mathrm{GHz}$ photoinjector [2], the longitudinal $\mathrm{rf}$ electric field is on the order of ( 90-100 MV/m). The transverse rf electric field measured at the beam radius is approximately $3 \%$ of the longitudinal electric field in this experiment, and using the beam parameters, such as bunch charge $Q \cong$ $0.96 \mathrm{nC}$, beam radius $r_{b} \cong 1 \mathrm{~mm}$, and bunch length $L \cong$ $3 \mathrm{~mm}$, one can roughly compute the magnitude of the transverse space-charge field to be $6 \%-10 \%$ of the longitudinal rf electric field near the cathode. Of course, the velocity ratio will be further reduced by a factor of $1 / \gamma$, where $\gamma$ is the relativistic beam factor, as the beam is accelerated by the longitudinal rf electric field. Since the velocity ratio is less than $10 \%$ for typical photoinjector systems, it is sufficient to approximate the beam currents for electromagnetic field calculations as $\mathbf{J}(\mathbf{r}, t)=$ $J_{z}(\mathbf{r}, t) \hat{\mathbf{e}}_{z}$.

\section{A. Arbitrary pipe cross section with longitudinal currents}

We assume that the cross section of the pipe is arbitrary, but uniform for $z>0$. For this system, the electromagnetic potentials $\phi(\mathbf{r}, t)$ and $\mathbf{A}(\mathbf{r}, t)=A_{z}(\mathbf{r}, t) \hat{\mathbf{e}}_{z}$, which yield the fields $\mathbf{E}(\mathbf{r}, t)=-\nabla \phi-\partial \mathbf{A} / \partial t$ and $\mathbf{B}(\mathbf{r}, t)=\nabla \times \mathbf{A}$, can be solved in the Lorentz gauge for a given charge density $\rho(\mathbf{r}, t)$ and longitudinal current density $\mathbf{J}(\mathbf{r}, t)=J_{z}(\mathbf{r}, t) \hat{\mathbf{e}}_{z}$, i.e.

$$
\left(\nabla^{2}-\frac{1}{c^{2}} \frac{\partial^{2}}{\partial t^{2}}\right) \phi(\mathbf{r}, t)=-\frac{\rho(\mathbf{r}, t)}{\varepsilon_{0}}
$$

and

$$
\left(\nabla^{2}-\frac{1}{c^{2}} \frac{\partial^{2}}{\partial t^{2}}\right) A_{z}(\mathbf{r}, t)=-\mu_{0} J_{z}(\mathbf{r}, t)
$$

As an aside, we note that the potentials satisfy the Lorentz gauge condition, i.e.

$$
\frac{1}{c^{2}} \frac{\partial \phi}{\partial t}+\nabla \cdot \mathbf{A}=0
$$

and the beam charge and current densities satisfy the continuity equation, i.e.

$$
\frac{\partial \rho}{\partial t}+\nabla \cdot \mathbf{J}=0
$$

At the perfectly conducting boundaries the fields must satisfy the conditions:

$$
\mathbf{E}(\mathbf{r}, t) \times\left.\hat{\mathbf{n}}\right|_{\text {surf }}=\left.\mathbf{B}(\mathbf{r}, t) \cdot \hat{\mathbf{n}}\right|_{\text {surf }}=0,
$$

where $\hat{\mathbf{n}}$ denotes the normal vector on the conductor surface. Equation (3) can be satisfied by specifying the bound- ary conditions for the potentials to be

$$
\begin{gathered}
\left.\phi\right|_{\text {surf }}=0, \\
\mathbf{A} \times\left.\hat{\mathbf{n}}\right|_{\text {surf }}=\left.A_{z}\right|_{\text {surf }} ^{z>0}=0, \\
\left.(\hat{\mathbf{n}} \cdot \nabla)(\mathbf{A} \cdot \hat{\mathbf{n}})\right|_{\text {surf }}=\left.\frac{\partial A_{z}}{\partial z}\right|_{\text {surf }} ^{z=0}=0 .
\end{gathered}
$$

We note that Eqs. (6) and (7) yield the correct boundary conditions for the fields, and Eq. (8) is obtained from Eqs. (6) and (7) along with the gauge condition in Eq. (3).

The electromagnetic potentials may be written in terms of two electromagnetic Green's functions $G_{\phi}\left(\mathbf{r}, t ; \mathbf{r}^{\prime}, t^{\prime}\right)$ and $G_{z}\left(\mathbf{r}, t ; \mathbf{r}^{\prime}, t^{\prime}\right)$ as

$$
\phi(\mathbf{r}, t)=\frac{1}{\varepsilon_{0}} \int_{-\infty}^{t} \int G_{\phi}\left(\mathbf{r}, t ; \mathbf{r}^{\prime}, t^{\prime}\right) \rho\left(\mathbf{r}^{\prime}, t^{\prime}\right) d^{3} \mathbf{r}^{\prime} d t^{\prime},
$$

and

$$
A_{z}(\mathbf{r}, t)=\mu_{0} \int_{-\infty}^{t} \int G_{z}\left(\mathbf{r}, t ; \mathbf{r}^{\prime}, t^{\prime}\right) J_{z}\left(\mathbf{r}^{\prime}, t^{\prime}\right) d^{3} \mathbf{r}^{\prime} d t^{\prime}
$$

where in the regime $z>0$ both Green's functions satisfy the inhomogeneous wave equation with a delta function source, i.e.

$$
\left(\nabla^{2}-\frac{1}{c^{2}} \frac{\partial^{2}}{\partial t^{2}}\right) G_{\phi, z}=-\delta\left(\mathbf{r}-\mathbf{r}^{\prime}\right) \delta\left(t-t^{\prime}\right)
$$

but are subject to different boundary conditions, namely

$$
\begin{array}{r}
\left.G_{\phi}\right|_{\text {surf }}=0, \\
\left.G_{z}\right|_{\text {surf }} ^{z>0}=0, \\
\left.\frac{\partial G_{z}}{\partial z}\right|_{\text {surf }} ^{z=0}=0 .
\end{array}
$$

Since the Green's functions which are defined by Eq. (10) are generated by delta functions in both space and time, it is possible to calculate the electromagnetic potentials, and hence, the electromagnetic fields for arbitrary beam charge and current densities as shown in Eqs. (9a) and (9b).

The solutions to $G_{\phi, z}$ can be constructed from the homogeneous solutions of Eq. (10), i.e.

$$
\begin{aligned}
G_{\phi} & =\frac{c \theta\left(t-t^{\prime}\right)}{2} \sum_{m n} \psi_{m n}\left(\mathbf{r}_{\perp}\right) \psi_{m n}^{*}\left(\mathbf{r}_{\perp}^{\prime}\right) \times\left[\Gamma_{-}-\Gamma_{+}\right] \\
G_{z} & =\frac{c \theta\left(t-t^{\prime}\right)}{2} \sum_{m n} \psi_{m n}\left(\mathbf{r}_{\perp}\right) \psi_{m n}^{*}\left(\mathbf{r}_{\perp}^{\prime}\right) \times\left[\Gamma_{-}+\Gamma_{+}\right],
\end{aligned}
$$

where $\mathbf{r}_{\perp}=\mathbf{r}-z \hat{\mathbf{e}}_{z}$ and $\mathbf{r}_{\perp}^{\prime}=\mathbf{r}^{\prime}-z^{\prime} \hat{\mathbf{e}}_{z}$ are transverse vectors, the normalized eigenfunctions $\psi_{m n}\left(\mathbf{r}_{\perp}\right)$ are the Dirichlet solutions of the transverse 2D Helmholtz equation with eigenvalues $k_{\perp m n}$, i.e. 


$$
\begin{aligned}
\left(\nabla^{2}+k_{\perp m n}^{2}\right) \psi_{m n}\left(\mathbf{r}_{\perp}\right) & =0, \\
\left.\psi_{m n}\left(\mathbf{r}_{\perp}\right)\right|_{\text {surf }} & =0, \\
\int\left|\psi_{m n}\left(\mathbf{r}_{\perp}\right)\right|^{2} d^{2} \mathbf{r}_{\perp} & =1,
\end{aligned}
$$

and

$$
\Gamma_{ \pm}=\frac{1}{\pi} \int_{-\infty}^{\infty} d k \frac{e^{i k\left(z \pm z^{\prime}\right)} \sin \left(c \sqrt{k_{\perp m n}^{2}+k^{2}}\left(t-t^{\prime}\right)\right)}{\sqrt{k_{\perp m n}^{2}+k^{2}}} .
$$

The Green's functions defined by the eigenfunction expansions in Eqs. (12a) and (12b), as well as the associated $\Gamma_{-}$and $\Gamma_{+}$defined by Eq. (14), can be readily obtained in the following manner. First, by using the fact that $\theta(t-$ $\left.t^{\prime}\right) \psi_{m n}\left(\mathbf{r}_{\perp}\right) e^{i k z}\left(C_{m n} \cos \left(\omega\left(t-t^{\prime}\right)\right)+S_{m n} \sin \left(\omega\left(t-t^{\prime}\right)\right)\right)$ forms a complete set of eigenfunctions which are solutions to the homogeneous 3D Helmholtz equation, where $C_{m n}$ and $S_{m n}$ are constants and $\omega=c \sqrt{k_{\perp m n}^{2}+k^{2}}$, we can express $G_{\phi, z}$ as a sum over these eigenfunctions. The factor $\theta\left(t-t^{\prime}\right)$ is included to ensure causality. By inserting the expansion for $G_{\phi, z}$ into Eq. (10) and then integrating both sides by $\int_{t^{\prime}-\varepsilon}^{t^{\prime}+\varepsilon} d t$ in the limit $\varepsilon \rightarrow 0$, it can be easily shown that $C_{m n}=0$ and $G_{\phi, z}$ and $\Gamma_{ \pm}$are given by Eqs. (12a), (12b), and (14). To be specific, the terms in $G_{\phi, z}$ corresponding to $\Gamma_{+}$yield an extra term proportional to $\delta(z+$ $\left.z^{\prime}\right)$ on the right-hand side of Eq. (10). However, since $z, z^{\prime}>0$, this term is zero. The factor $\Gamma_{+}$in Eqs. (12a) and $(12 b)$ is necessary to satisfy the cathode boundary conditions at $z=0$ given by Eqs. (11a) and (11b). From a physical point of view, $\Gamma_{-}$and $\Gamma_{+}$, represent the contributions of the real charge and image charge (due to the cathode at $z=0$ ), respectively, on the electromagnetic fields. We note that the method of constructing Green's functions from eigenfunctions is a well-known method that is used extensively for solving nonhomogeneous scalar wave equations [10].

The integral in Eq. (14) may be simplified [11] to

$$
\Gamma_{ \pm}=J_{0}\left(k_{\perp m n} \lambda_{ \pm}\right) \theta\left(\lambda_{ \pm}^{2}\right),
$$

where $J_{0}(x)$ is the zeroth order Bessel function of the first kind, $\theta(x)$ is the step function, and

$$
\lambda_{ \pm}^{2}=c^{2}\left(t-t^{\prime}\right)^{2}-\left(z \pm z^{\prime}\right)^{2} .
$$

Using Eq. (4) and integration by parts, one can readily check that the solutions given by Eqs. (9) and (12) satisfy the Lorentz gauge condition. We note that the factors $\theta\left(\lambda_{-}^{2}\right)$ and $\theta\left(\lambda_{+}^{2}\right)$ enforce a causality condition on the electromagnetic waves emanating from the beam charge and induced image charges, respectively. When analyzing the potentials near the front of the bunch, these factors allow for rapid numerical convergence since only sources "sufficiently close" to the point of observation need to be considered.

The electromagnetic fields $\mathbf{E}(\mathbf{r}, t)$ and $\mathbf{B}(\mathbf{r}, t)$ can be computed from the potentials in Eq. (9). In general, the electric field will have components in both the longitudinal and transverse directions, but under the assumption of only longitudinal beam currents, the magnetic field will only have components in the transverse directions. The transverse electric and magnetic fields follow immediately from Eq. (9), and are given by

$$
\begin{aligned}
\mathbf{E}_{\perp}(\mathbf{r}, t)= & -\frac{c}{2 \varepsilon_{0}} \sum_{m n} \nabla_{\perp} \psi_{m n}\left(\mathbf{r}_{\perp}\right) \int_{-\infty}^{t} \int \psi^{*}{ }_{m n}\left(\mathbf{r}_{\perp}^{\prime}\right) \\
& \times\left[\Gamma_{-}-\Gamma_{+}\right] \rho\left(\mathbf{r}^{\prime}, t^{\prime}\right) d^{3} \mathbf{r}^{\prime} d t^{\prime}
\end{aligned}
$$

and

$$
\begin{aligned}
\mathbf{B}_{\perp}(\mathbf{r}, t)= & -\frac{\mu_{0}}{2} \sum_{m n} \hat{\mathbf{e}}_{z} \times \nabla_{\perp} \psi_{m n}\left(\mathbf{r}_{\perp}\right) \int_{-\infty}^{t} \int \psi_{m n}^{*}\left(\mathbf{r}_{\perp}^{\prime}\right) \\
& \times\left[\Gamma_{-}+\Gamma_{+}\right] J_{z}\left(\mathbf{r}^{\prime}, t^{\prime}\right) d^{3} \mathbf{r}^{\prime} d t^{\prime}
\end{aligned}
$$

The final solution to the electric field in the longitudinal direction requires a few mathematical steps. To start,

$$
\begin{aligned}
E_{z}= & -\frac{1}{\varepsilon_{0}} \int_{-\infty}^{t} \int d^{3} \mathbf{r}^{\prime} d t^{\prime}\left[\frac{\partial G_{\phi}}{\partial z} \rho+\frac{1}{c^{2}} \frac{\partial G_{z}}{\partial t} J_{z}\right] \\
= & -\frac{c}{2 \varepsilon_{0}} \int d^{2} \mathbf{r}_{\perp}^{\prime} \sum_{m n} \psi_{m n}\left(\mathbf{r}_{\perp}\right) \psi^{*}{ }_{m n}\left(\mathbf{r}_{\perp}^{\prime}\right) \int_{-\infty}^{t} d t^{\prime} \\
& \times \int d z^{\prime}\left[\left(\frac{\partial \lambda_{-}}{\partial z} \frac{\partial \Gamma_{-}}{\partial \lambda_{-}}-\frac{\partial \lambda_{+}}{\partial z} \frac{\partial \Gamma_{+}}{\partial \lambda_{+}}\right) \rho\right. \\
& \left.+\left(\frac{\partial \lambda_{-}}{\partial t} \frac{\partial \Gamma_{-}}{\partial \lambda_{-}}+\frac{\partial \lambda_{+}}{\partial t} \frac{\partial \Gamma_{+}}{\partial \lambda_{+}}\right) \frac{J_{z}}{c^{2}}\right] .
\end{aligned}
$$

Then using the fact that $d J_{0}(x) / d x=-J_{1}(x)$,

$$
\frac{d \theta\left(\lambda_{ \pm}^{2}\right)}{d \lambda_{ \pm}}=2 \lambda_{ \pm} \delta\left(\lambda_{ \pm}^{2}\right)=\lambda_{ \pm} \frac{\delta\left(z^{\prime} \pm\left(z+c\left(t-t^{\prime}\right)\right)\right)+\delta\left(z^{\prime} \pm\left(z-c\left(t-t^{\prime}\right)\right)\right)}{\left|z \pm z^{\prime}\right|}
$$

and the completeness relation,

$$
\sum_{m n} \psi_{m n}\left(\mathbf{r}_{\perp}\right) \psi_{m n}^{*}\left(\mathbf{r}_{\perp}^{\prime}\right)=\delta\left(\mathbf{r}_{\perp}-\mathbf{r}_{\perp}^{\prime}\right)
$$

we can express the longitudinal electric field as 


$$
\begin{aligned}
E_{z}= & \frac{c}{2 \varepsilon_{0}} \int d^{2} \mathbf{r}_{\perp}^{\prime} \sum_{m n} k_{\perp m n} \psi_{m n}\left(\mathbf{r}_{\perp}\right) \psi^{*}{ }_{m n}\left(\mathbf{r}_{\perp}^{\prime}\right) \int_{-\infty}^{t} d t^{\prime} \int d z^{\prime}\left\{\frac{\theta\left(\lambda_{-}^{2}\right)}{\lambda_{-}} J_{1}\left(k_{\perp m n} \lambda_{-}\right)\left[-\left(z-z^{\prime}\right) \rho+\frac{J_{z}\left(t-t^{\prime}\right)}{c}\right]\right. \\
& \left.+\frac{\theta\left(\lambda_{+}^{2}\right)}{\lambda_{+}} J_{1}\left(k_{\perp m n} \lambda_{+}\right)\left[\left(z+z^{\prime}\right) \rho+\frac{J_{z}\left(t-t^{\prime}\right)}{c}\right]\right\}+\frac{c}{2 \varepsilon_{0}} \int_{-\infty}^{t} d t^{\prime}\left\{\theta ( z - c ( t - t ^ { \prime } ) ) \left[\rho\left(\mathbf{r}_{\perp}, z-c\left(t-t^{\prime}\right), t^{\prime}\right)\right.\right. \\
& \left.-J_{z}\left(\mathbf{r}_{\perp}, z-c\left(t-t^{\prime}\right), t^{\prime}\right) / c\right]-\theta\left(z+c\left(t-t^{\prime}\right)\right)\left[\rho\left(\mathbf{r}_{\perp}, z+c\left(t-t^{\prime}\right), t^{\prime}\right)+J_{z}\left(\mathbf{r}_{\perp}, z+c\left(t-t^{\prime}\right), t^{\prime}\right) / c\right] \\
& \left.-\theta\left(-z+c\left(t-t^{\prime}\right)\right)\left[\rho\left(\mathbf{r}_{\perp},-z+c\left(t-t^{\prime}\right), t^{\prime}\right)+J_{z}\left(\mathbf{r}_{\perp},-z+c\left(t-t^{\prime}\right), t^{\prime}\right) / c\right]\right\} .
\end{aligned}
$$

We note that the step functions found in the last integral term arise from the fact that the delta functions in Eq. (20) will only yield finite contributions after integration in $z^{\prime}$ when the zeros of the delta functions occur for $z^{\prime}>0$. Since it is always true that $-z-c\left(t-t^{\prime}\right)<0$, the term which is proportional to $\theta\left(-z-c\left(t-t^{\prime}\right)\right)$ does not contribute to the last term in Eq. (22).

For the special case of a circular pipe with radius $a$, the transverse eigenfunctions and corresponding eigenvalues which solve Eq. (13) are given in cylindrical coordinates by

$$
\psi_{m n}\left(\mathbf{r}_{\perp}\right)=\frac{1}{a \sqrt{\pi}} \frac{J_{m}\left(j_{m n} r / a\right) e^{i m \theta}}{\left|J_{m+1}\left(j_{m n}\right)\right|}
$$

and

$$
k_{\perp m n}=j_{m n} / a,
$$

where $J_{m}(x)$ is the $m$ th order Bessel function and $j_{m n}$ is the $n$th positive root of $J_{m}(x)$. Hence, Eq. (23) along with Eqs. (17), (18), and (22) yield a complete description for computing the space-charge fields in a circular pipe with a cathode at $z=0$. We should emphasize that the cylindrical symmetry necessary for this solution is only placed on the pipe, but not on the charge and current distributions. Therefore, it is possible to use this formulation to simulate the electromagnetic fields for arbitrary longitudinal beam currents, such as those with dipole and quadrupole moments, within a cylindrical pipe.

In the future, the authors are planning to expand the analysis presented in this section by including the effect of one or more metallic irises on space-charge fields. Irises are typically found in photoinjector systems, and the first iris along with the cathode and cavity walls usually define the boundary of the first half-cell in a photoinjector. The irises will affect the space-charge fields with the presence of additional image charges and image currents. There are a variety of methods which can be utilized for including the effects of irises. For example, in Refs. [8,9], the authors expanded the space-charge fields in each region of injector space, i.e., before and after the iris, using eigenfunctions which locally satisfy the correct boundary conditions. The coefficients of the field expansions were found numerically by applying boundary conditions, such as field continuity, at the location of the iris. Usually, this technique results in determining the elements of a large $N \times M$ matrix where $N$ and $M$ are the number of eigenmodes used in expanding the fields before and after the iris. Another technique with which the present authors are actively pursuing is a method developed by Bethe [12] that expands the fields due to the iris(es) using a perturbative multipole field expansion. This technique starts by solving the space-charge fields assuming that no iris is present, i.e., that the geometry of the conductor is a pillbox. These lowest order electromagnetic fields can then be used to compute the induced electric and magnetic multipole moments of the iris to all orders in $b / a$, where the iris radius is $b$ and the cavity radius is the previously defined pipe radius, $a$. The total space-charge field can then be represented as the zeroth order fields plus the fields due to the electric and magnetic multipoles for each iris.

\section{B. Rectangular pipe with arbitrary currents}

We now show the exact solution to the electromagnetic potentials when the pipe in Fig. 1 has a rectangular cross section. In this system, it is possible to find the explicit potentials for the more general assumption of arbitrary beam currents, that is currents which have both longitudinal as well as transverse components. We denote the sides of the pipe by $x=0, x=L, y=0$, and $y=W$. As in the previous case, the electromagnetic potentials $\phi(\mathbf{r}, t)$ and $\mathbf{A}(\mathbf{r}, t)$, may be solved in the Lorentz gauge. The difference in this case, however, is that $\mathbf{A}(\mathbf{r}, t)$ will have all three components instead of only the longitudinal component as in the previous case. For a given $\rho(\mathbf{r}, t)$ and $\mathbf{J}(\mathbf{r}, t)$ which satisfy Eq. (4), the potentials are given by

$$
\left(\nabla^{2}-\frac{1}{c^{2}} \frac{\partial^{2}}{\partial t^{2}}\right) \phi(\mathbf{r}, t)=-\frac{\rho(\mathbf{r}, t)}{\varepsilon_{0}}
$$

and

$$
\left(\nabla^{2}-\frac{1}{c^{2}} \frac{\partial^{2}}{\partial t^{2}}\right) \mathbf{A}(\mathbf{r}, t)=-\mu_{0} \mathbf{J}(\mathbf{r}, t)
$$

and satisfy the boundary conditions listed in Eqs. (6)-(8).

Similar to the previous case, the solutions to the electromagnetic potentials can be expressed in terms of four time-dependent Green's functions, which we denote by $G_{i}\left(\mathbf{r}, t ; \mathbf{r}^{\prime}, t^{\prime}\right)$ where $i=\phi, x, y$, and $z$. The potentials are given by

$$
\phi(\mathbf{r}, t)=\frac{1}{\varepsilon_{0}} \int_{-\infty}^{t} \int G_{\phi}\left(\mathbf{r}, t ; \mathbf{r}^{\prime}, t^{\prime}\right) \rho\left(\mathbf{r}^{\prime}, t^{\prime}\right) d^{3} \mathbf{r}^{\prime} d t^{\prime}
$$


and

$$
A_{\alpha}(\mathbf{r}, t)=\mu_{0} \int_{-\infty}^{t} \int G_{\alpha}\left(\mathbf{r}, t ; \mathbf{r}^{\prime}, t^{\prime}\right) J_{\alpha}\left(\mathbf{r}^{\prime}, t^{\prime}\right) d^{3} \mathbf{r}^{\prime} d t^{\prime},
$$

where $\alpha=x, y$, and $z$. All four Green's functions satisfy the differential equation

$$
\left(\nabla^{2}-\frac{1}{c^{2}} \frac{\partial^{2}}{\partial t^{2}}\right) G_{i}=-\delta\left(\mathbf{r}-\mathbf{r}^{\prime}\right) \delta\left(t-t^{\prime}\right),
$$

and are subject to the following boundary conditions, namely

$$
\begin{array}{r}
\left.G_{\phi}\right|_{\text {surf }}=0, \\
\left.G_{x}\right|_{y=0, y=W}=\left.\frac{\partial G_{x}}{\partial x}\right|_{x=0, x=L}=0, \\
\left.G_{y}\right|_{x=0, x=L}=\left.\frac{\partial G_{y}}{\partial y}\right|_{y=0, y=W}=0, \\
\left.G_{z}\right|_{\substack{x=0, x=L \\
y=0, y}}=\left.\frac{\partial G_{z}}{\partial z}\right|_{z=0}=0 .
\end{array}
$$

The solutions to $G_{i}$ can be readily constructed in a fashion similar to the previous case, i.e.

$$
\begin{aligned}
G_{\phi}= & \frac{2 c}{L W} \sum_{m=1}^{\infty} \sum_{n=1}^{\infty} \sin \left(\frac{m \pi x}{L}\right) \sin \left(\frac{m \pi x^{\prime}}{L}\right) \sin \left(\frac{n \pi y}{W}\right) \\
& \times \sin \left(\frac{n \pi y^{\prime}}{W}\right)\left[\Gamma_{-}-\Gamma_{+}\right], \\
G_{x}= & \frac{2 c}{L W} \sum_{m=1}^{\infty} \sum_{n=1}^{\infty} \cos \left(\frac{m \pi x}{L}\right) \cos \left(\frac{m \pi x^{\prime}}{L}\right) \sin \left(\frac{n \pi y}{W}\right) \\
& \times \sin \left(\frac{n \pi y^{\prime}}{W}\right)\left[\Gamma_{-}-\Gamma_{+}\right], \\
G_{y}= & \frac{2 c}{L W} \sum_{m=1}^{\infty} \sum_{n=1}^{\infty} \sin \left(\frac{m \pi x}{L}\right) \sin \left(\frac{m \pi x^{\prime}}{L}\right) \cos \left(\frac{n \pi y}{W}\right) \\
& \times \cos \left(\frac{n \pi y^{\prime}}{W}\right)\left[\Gamma_{-}-\Gamma_{+}\right], \\
G_{z}= & \frac{2 c}{L W} \sum_{m=1}^{\infty} \sum_{n=1}^{\infty} \sin \left(\frac{m \pi x}{L}\right) \sin \left(\frac{m \pi x^{\prime}}{L}\right) \sin \left(\frac{n \pi y}{W}\right) \\
& \times \sin \left(\frac{n \pi y^{\prime}}{W}\right)\left[\Gamma_{-}+\Gamma_{+}\right],
\end{aligned}
$$

where

$$
k_{\perp m n}=\sqrt{\left(\frac{m \pi}{L}\right)^{2}+\left(\frac{n \pi}{W}\right)^{2}} .
$$

The reason why it is relatively straightforward to construct the potential solutions in this geometry for arbitrary beam currents as opposed to a general curvilinear pipe, such as a circular pipe, is due to two reasons. First, in the rectangular case for which it is appropriate to utilize
Cartesian coordinates, Eq. (24b) can be separated into three component equations. In these equations, there is no coupling between the two transverse directions, i.e. $J_{x}$ generates $A_{x}$ but not $A_{y}$, and vice versa. This is not the case, however, in general curvilinear coordinate systems, such as cylindrical coordinates, in which both $A_{r}$ and $A_{\theta}$ are generated when either $J_{r}$ or $J_{\theta}$ are present. Second, in order to satisfy the Lorentz gauge condition with the potentials, it is necessary to change the order of differentiation in the eigenfunctions from unprimed coordinates to prime coordinates using integration by parts, and then to exploit the continuity equation. This was readily accomplished for longitudinal currents when the eigenfunctions in the longitudinal direction and in time are sinusoidal as in Eq. (14). Since the transverse eigenfunctions for the rectangular case are also given by sinusoidal functions, the gauge condition can be readily satisfied for the transverse potentials as well.

\section{NUMERICAL IMPLEMENTATION AND BENCHMARKING}

We now briefly describe how the electromagnetic fields given in Eqs. (17), (18), and (22) can be numerically solved, and then show results of a benchmarking study which was used to validate the accuracy of the numerical scheme. The authors will be addressing all of the computational requirements necessary for accurately computing the fields to within less than $1 \%$ error in a later paper [13].

In order to evaluate the fields numerically from Eqs. (17), (18), and (22), it is necessary to perform three distinct operations: a double series over the transverse eigenfunctions, a time integration, and a spatial integration over all three coordinates. Hence, in order to determine the fields accurately for a given simulation, it is essential to understand the requirements on the following numerical parameters: (i) the number of transverse modes, (ii) the integration time step, and (iii) the spatial integration steps in all three directions.

In general, the convergence rate of the series, and hence the number of modes necessary, will strongly depend on the transverse size of the beam. Typically, the number of transverse modes necessary for accurately resolving the fields will be inversely proportional to the transverse size of the beam. A good rule for figuring out the number of modes necessary to achieve a certain level of accuracy in the fields is to find the number of modes necessary for achieving at least the same level of accuracy when expanding the charge density in terms of the eigenfunctions, $\psi_{m n}\left(\mathbf{r}_{\perp}\right)$, i.e.

$$
\rho(\mathbf{r}, t)=\sum_{m n} \rho_{m n}(z, t) \psi_{m n}\left(\mathbf{r}_{\perp}\right),
$$

where $\rho_{m n}(z, t)$ are expansion coefficients. In the specific case of a cylindrically symmetric beam with a characteristic beam radius $r_{b}$ inside of a circular pipe of radius $a$, the typical number of radial modes, $M$, necessary for modeling 
the fields to within $1 \%$ error is given by

$$
M \geq \frac{40 a}{r_{b}} .
$$

The integration time step, $\Delta t^{\prime}$, needs to be sufficiently small for two distinct reasons - one which is based on beam dynamics and another which is based on the error of the numerical integration. From the beam dynamics point of view, $\Delta t^{\prime}$ needs to be smaller than any characteristic time scale related to beam motion in order to resolve the fields associated with the beam motion. For example, if a bunch of electrons is accelerated in the source by an external electric field, $E_{\text {ext }}$, and achieves a relativistic energy factor, $\gamma$, then the dynamical time scale in the system according to the Lorentz force law is given by $\tau_{\mathrm{dyn}} \sim \gamma m_{e} c / e E_{\mathrm{ext}}$, where $m_{e}$ is the mass of the electron. A completely different limit on $\Delta t^{\prime}$ emerges, however, when considering the numerical errors due to time integration. The integrands of the field equations contain Bessel functions, $J_{0}\left(k_{\perp m n} \lambda_{ \pm}\right)$, which will typically oscillate between positive and negative values either one or multiple times as the parameter $t^{\prime}$ is varied within the limits of integration, i.e. $-\infty \leq t^{\prime} \leq t$. Hence, in order to accurately perform the time integration, $\Delta t^{\prime}$ must be sufficiently small compared to the oscillation time of the Bessel function corresponding to the highest order transverse mode used in the series expansion. Since the highest order mode number is inversely proportional to the beam size as in Eq. (31), it can be shown that $\Delta t^{\prime}$ should be proportional to the beam size. In particular, for a cylindrically symmetric beam, it is found that setting

$$
\Delta t^{\prime} \ll \frac{0.01 r_{b}}{c}
$$

yields excellent results for the field computation. We note that any electromagnetic waves generated at the center of the bunch at $r=0$ will take at least a time $r_{b} / c$ to propagate through the bunch. Hence, Eq. (32) ensures that any effects involving waves propagating through the bunch will be modeled accurately. When simulating photocathode electron sources, for example, it is usually the case that the time scale shown in Eq. (32) is much smaller than $\tau_{\text {dyn }}$. Hence, Eq. (32) will typically be appropriate for computing the space-charge fields in a photocathode source simulation.

Finally, it is obvious that if $\rho(\mathbf{r}, t)$ and $\mathbf{J}(\mathbf{r}, t)$ have characteristic length scales, then the spatial integration step sizes, $\Delta x^{\prime}, \Delta y^{\prime}$, and $\Delta z^{\prime}$ need to be sufficiently small compared to those scales in order to accurately resolve the fields. In the benchmark example that is illustrated in this section, we specify the distribution function of the beam for all times in the simulation, and the spatial integrations within Eqs. (17), (18), and (22) are carried out analytically. Hence, we do not need to specify the spatial integration step sizes for this example.
For beam simulations in which $\rho(\mathbf{r}, t)$ and $\mathbf{J}(\mathbf{r}, t)$ are not specified, but rather are time evolving due to the dynamics of the system macroparticles, it would be necessary to make the integration step sizes small enough to accurately compute $\rho(\mathbf{r}, t)$ and $\mathbf{J}(\mathbf{r}, t)$. One simple method for finding $\rho(\mathbf{r}, t)$ and $\mathbf{J}(\mathbf{r}, t)$ would be to divide the entire space into cells, and then to count the total charge and current in each cell. In this case, the spatial integration step sizes would be the cell sizes.

It is essential when developing a numerical algorithm to find benchmarks by which the algorithm may be compared. The benchmark that we now present is based on an analytical result. In particular, we test the case of a source with cylindrical pipe of radius $a$ whereby a bunch of total charge $Q$ is emitted from the cathode at time $t=0$ with a constant uniform velocity $\mathbf{V}=V \hat{\mathbf{e}}_{z}$. The bunch is assumed to be cylindrically symmetric, have zero longitudinal length, and have finite size in the transverse directions. We assume that the transverse radial density of the bunch is parabolic with radius $r_{b}$ and fixed in time. The charge and current densities are given by

$$
\rho(\mathbf{r}, t)=\rho_{\perp}\left(\mathbf{r}_{\perp}\right) \delta(z-V t)
$$

and

$$
J_{z}(\mathbf{r}, t)=\rho_{\perp}\left(\mathbf{r}_{\perp}\right) V \delta(z-V t) .
$$

where $\rho_{\perp}\left(\mathbf{r}_{\perp}\right)=\left(2 Q / \pi r_{b}^{2}\right) \theta\left(r_{b}-r\right)\left(1-r^{2} / r_{b}^{2}\right)$.

Now we compare the fields of this system to the fields of another system where there are two bunches $Q_{1}=Q$ and $Q_{2}=-Q$, with velocities $\mathbf{V}_{1}=V \hat{\mathbf{e}}_{z}$ and $\mathbf{V}_{2}=-\mathbf{V}_{1}$, respectively. The trajectories of the two bunches are configured, such that at time $t=0$, the two bunches will overlap . The charge and current densities of this second system are given by

$$
\begin{aligned}
& \rho_{1}(\mathbf{r}, t)=\rho_{\perp}\left(\mathbf{r}_{\perp}\right) \delta(z-V t), \\
& J_{z 1}(\mathbf{r}, t)=\rho_{\perp}\left(\mathbf{r}_{\perp}\right) V \delta(z-V t), \\
& \rho_{2}(\mathbf{r}, t)=-\rho_{\perp}\left(\mathbf{r}_{\perp}\right) \delta(z+V t),
\end{aligned}
$$

and

$$
J_{z 2}(\mathbf{r}, t)=\rho_{\perp}\left(\mathbf{r}_{\perp}\right) V \delta(z+V t) .
$$

The fields for each bunch in the second system can be easily computed by finding the electric field in the rest frame of the bunch, and then Lorentz transforming back to the laboratory frame. In the second system, the bunch $Q_{2}$ represents the image bunch which is found in the first system due to the presence of the cathode at $z=0$. For specific regions of space and time, e.g., before wave reflection from the pipe has occurred $(t<2 a / c$ for $r=0)$ and after the initial electromagnetic shock front due to bunch emission has passed ( $z<c t$ for $r=0$ ), the electromagnetic fields produced by the first system will be exactly the same as those of the second system. We note that, in the first system, the fields should be exactly zero when $z>c t$, 


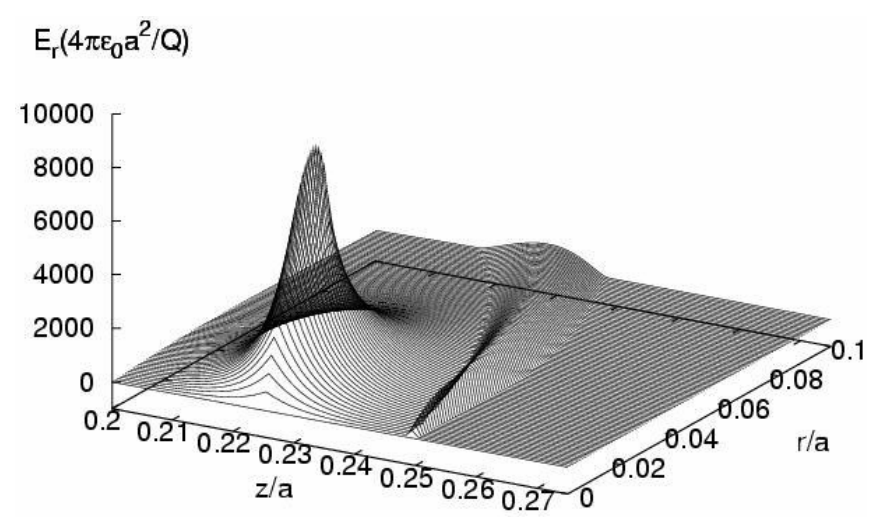

FIG. 2. Plot of $E_{r}\left(4 \pi \varepsilon_{0} a^{2}\right) / Q$ vs $r / a$ and $z / a$ for $t=0.25 a / c$.

since the causality condition prevents any waves from propagating beyond $z=c t$.

Figures 2-5 show plots of the normalized radial and longitudinal electric fields using the beam densities given in Eq. (33) and the field equations given by Eqs. (17) and (22). In the simulation, the following parameters were chosen: the beam velocity is $V=0.9 c$, the beam radius corresponds to the experimental beam radius in the $\mathrm{BNL}$ $2.856 \mathrm{GHz}$ photocathode experiment [2], i.e. $r_{b} / a=$ 0.0243 , and the time is $t=0.25 a / c$. In order to accurately compute the fields in accordance with Eqs. (31) and (32), we set $M=2000$ and $\Delta t^{\prime}=10^{-6} a / c$.

Figure 2 shows a 3D plot of $E_{r}\left(4 \pi \varepsilon_{0} a^{2}\right) / Q$ vs $r / a$ and $z / a$. There are two distinguishing features in Fig. 2-a large pulse near $z / a=0.225$, which is the bunch's axial location and a second smaller pulse with an outer edge at $z / a=0.25$. The pulse at $z / a=0.225$ represents the local electric field generated by the beam, while the second pulse represents the initial electromagnetic waves which were generated at $t=0$ when the bunch was emitted. Since the time in Fig. 2 is $t=0.25 a / c$, the point $z / a=0.25$ corre-

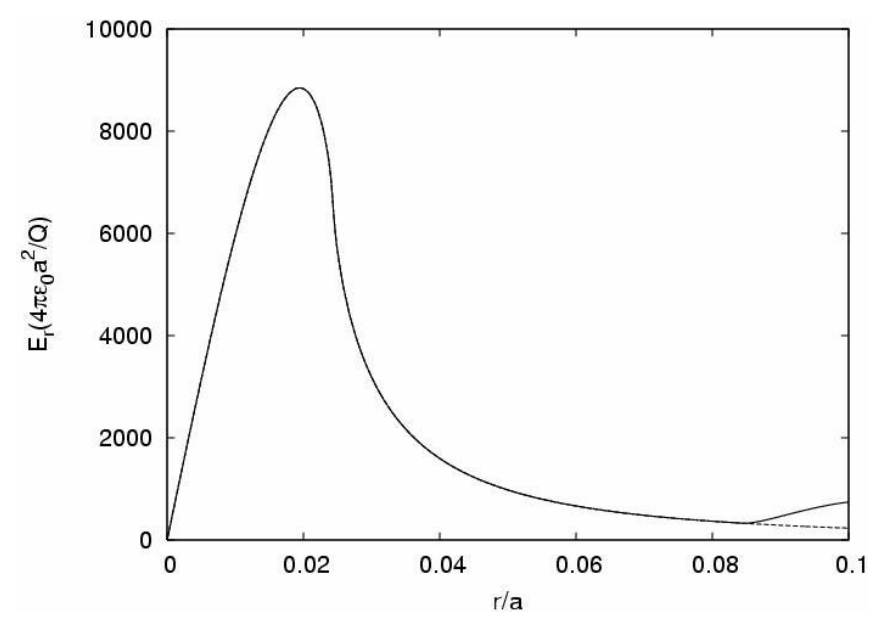

FIG. 3. Plots of $E_{r}\left(4 \pi \varepsilon_{0} a^{2}\right) / Q$ vs $r / a$ at the bunch location, $z / a=0.225$, showing the numerical scheme (solid line) and the benchmark example (dashed line).

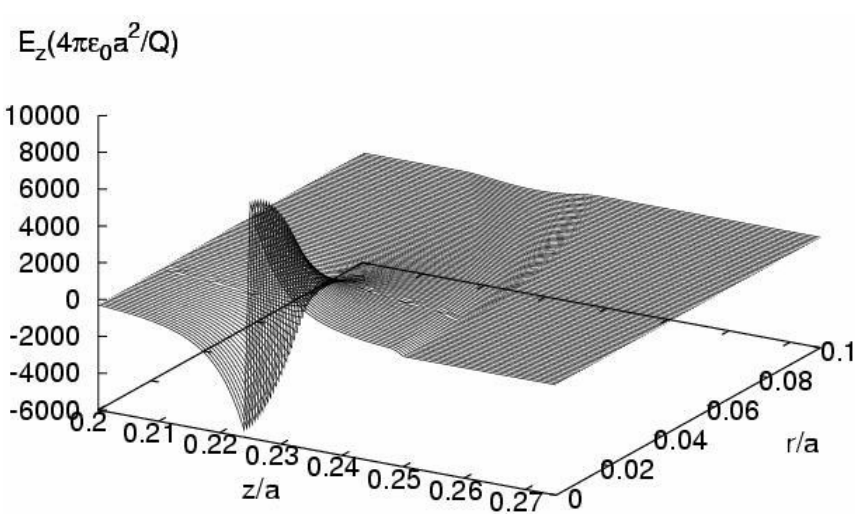

FIG. 4. Plot of $E_{z}\left(4 \pi \varepsilon_{0} a^{2}\right) / Q$ vs $r / a$ and $z / a$ for $t=0.25 a / c$.

sponds to the location of the causality condition, $z=c t$. From Fig. 2, it is also seen that the fields are zero when $z>c t$, which illustrates the effect of the causality condition. Figure 3 shows a plot (solid line) of $E_{r}\left(4 \pi \varepsilon_{0} a^{2}\right) / Q$ vs $r / a$ at the bunch's location $z / a=0.225$. In addition, a plot of the normalized radial electric field from the second (benchmark) system is shown (dashed line), which uses the densities in Eqs. (34) to compute the fields. From Fig. 3, it is seen that there is excellent agreement, i.e., less than $1 \%$ error, between the numerical scheme and the benchmark example for $r / a \leq 0.085$. The difference between the fields in Fig. 3 for $r / a \geq 0.085$ is attributed to the initial electromagnetic pulse generated at bunch emission. Essentially, one can view this transient electromagnetic pulse as being due to transition radiation, which occurs when the electron bunch is spontaneously generated at the cathode surface at a time $t=0$. Obviously, this electromagnetic pulse would not be present in the benchmark example since the two bunches defined by Eq. (34) are uniformly moving for all time. It is therefore necessary to wait a sufficient amount of time for the initial transient

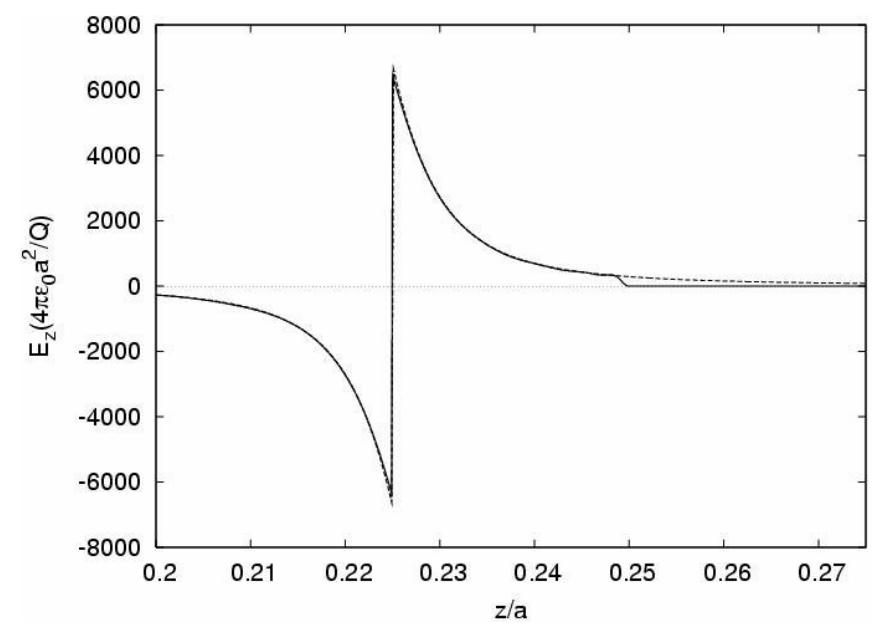

FIG. 5. Plots of $E_{z}\left(4 \pi \varepsilon_{0} a^{2}\right) / Q$ vs $z / a$ for $r=0$, showing the numerical scheme (solid line) and the benchmark example (dashed line). 
pulse to pass in order for the fields in the benchmark case and the numerical case to match.

Figure 4 shows a plot of $E_{z}\left(4 \pi \varepsilon_{0} a^{2}\right) / Q$ vs $r / a$ and $z / a$. Figure 4 shows similar features as Fig. 2, namely, two pulses - one near the beam and one generated by the initial waves due to bunch emission, i.e., the transient electromagnetic pulse due to transition radiation. The sharp discontinuity in the pulse near the beam is located precisely at the bunch's location, and denotes the point at which the sign of the electric field changes. Note that in Fig. 4, the field near the second pulse shows a trough instead of a crest which is shown in Fig. 2. The reason for this is that, since there is no charge density near the location of the second pulse, Maxwell's equations yield $\nabla \cdot \mathbf{E}(\mathbf{r}, t)=0$. Hence, the longitudinal electric field is decreasing while the radial component is increasing. Figure 5 shows a plot of $E_{z}\left(4 \pi \varepsilon_{0} a^{2}\right) / Q$ vs $z / a$ at $r=0$ for both the numerical scheme (solid line) as well as the benchmark example (dashed line). We see excellent agreement, i.e., less than $1 \%$ error between the fields for locations which are not close to the second pulse at $z=c t$. For $z>c t$, the numerical scheme correctly shows that the fields are zero according to the causality condition.

\section{SUMMARY}

In this paper, we have formulated and demonstrated a numerical scheme based on Green's function methods for computing the electromagnetic fields within a simplified electron source geometry. The conductor geometry consisted of a flat cathode at $z=0$ along with a uniform pipe which is semi-infinite in extent. We explicitly showed how to compute the fields for two cases, namely, a uniform pipe with arbitrary cross section and longitudinal beam currents and a rectangular pipe with arbitrary beam currents. The Green's functions within this scheme are constructed from eigenfunctions which exactly satisfy the boundary conditions at the conductor surfaces. In addition, these functions include the necessary causality constraints, i.e. $\theta\left(\lambda_{ \pm}^{2}\right)$, which are due to the finite speed of light. For any given $\rho(\mathbf{r}, t)$ and $\mathbf{J}(\mathbf{r}, t)$ which satisfy the continuity equation, the electromagnetic potentials in the Lorentz gauge, and hence, the space-charge fields, $\mathbf{E}(\mathbf{r}, t)$ and $\mathbf{B}(\mathbf{r}, t)$, can be computed which satisfy both the correct boundary conditions, as well as, the correct causality constraints.

Using an analytical benchmark case, we have shown the high level of accuracy, less than 1\% error in the fields, which is possible with the Green's function scheme. A more detailed study of the relatively small error associated with the fields in the numerical scheme will be presented in a later paper [13]. The benchmark example also illustrates how the scheme can resolve other interesting phenomena, such as image charge effects due to the cathode surface as well as the transient electromagnetic effects which are generated by the beam emission.

The authors plan to continue developing the Green's function scheme for future simulation studies of electron sources. In particular, we are actively working to develop methods for computing the fields within curvilinear pipes when transverse currents are present. In addition, we are working on techniques for including the effects of more complicated conducting structures, such as irises, which are typically present in many electron sources including photocathode sources.

\section{ACKNOWLEDGMENTS}

This research was supported in part by the Department of Energy under Grant No. DE-FG0292ER40747 and in part by the National Science Foundation under Grant No. PHY-0552389 and the REU program.

[1] G. Suberlucq, in Proceedings of the 2004 European Particle Accelerator Conference, Lucerne, Switzerland (EPS-AG/CERN, Geneva, 2004), p. 64.

[2] K. Batchelor, H. Kirk, J. Sheehan, M. Woodle, and K. McDonald, in Proceedings of the 1988 European Particle Accelerator Conference, Rome, Italy (EPS-AG/ CERN, Geneva, 1988), p. 954.

[3] L. M. Young, Report No. LA-UR-96-1835, Los Alamos, 1996, documentation by J. H. Billen (rev. 2004).

[4] L. Gianessi et al., Nucl. Instrum. Methods Phys. Res., Sect. A 436, 443 (1999).

[5] MAFIA Collaboration, MAFIA Manual (CST GmbH, Darmstadt, 1997).

[6] K.S. Yee, IEEE Trans. Antennas Propag. 14, 302 (1966).

[7] I. Zagorodnov and T. Weiland, Phys. Rev. ST Accel. Beams 8, 042001 (2005).

[8] W. Salah and J. M. Dolique, Nucl. Instrum. Methods Phys. Res., Sect. A 447, 309 (2000).

[9] W. Salah, Nucl. Instrum. Methods Phys. Res., Sect. A 533, 248 (2004).

[10] See, for example, G. Barton, Elements of Green's Functions and Propagation: Potentials, Diffusion, and Waves (Oxford University Press, New York, 1989), Chap. 10.

[11] I.S. Gradshteyn and I. M. Ryzhik, Table of Integrals, Series, and Products, 6th ed. (Academic, London, 2000), p. 476.

[12] H. A. Bethe, Phys. Rev. 66, 163 (1944).

[13] C. S. Park and M. Hess (unpublished). 